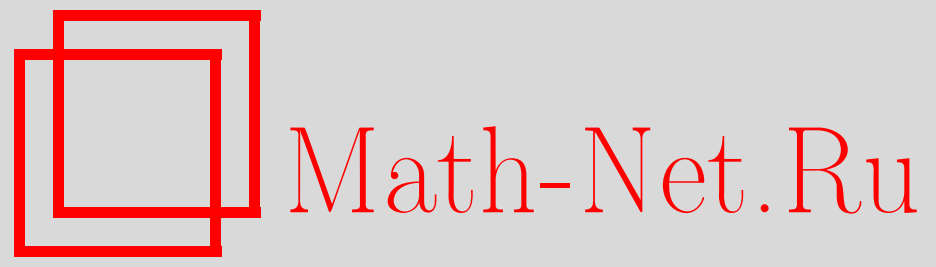

А. Лаптев, Отрицательный спектр класса двумерных операторов Шрёдингера с потенциалами, зависящими от радиуса, Функи. анализ и его прил., 2000, том 34, выпуск 4, 85-87

DOI: https://doi.org/10.4213/faa331

Использование Общероссийского математического портала MathNet.Ru подразумевает, что вы прочитали и согласны с пользовательским соглашением

http://www . mathnet.ru/rus/agreement

Параметры загрузки:

IP : 3.82 .47 .9

26 апреля 2023 г., 16:40:03

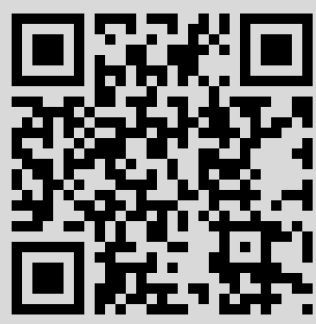


УДК 517.9

\title{
Отрицательный спектр класса двумерных операторов Шрёдингера с потенциалами, зависящими от радиуса
}

\author{
(C) 2000. А. ЛАПтеВ
}

1. Введение и основной результат. Предлагаемая заметка посвящается изучению отрицательного спектра самосопряженного оператора Шрёдингера, действующего в $L^{2}\left(\mathbb{R}^{d}\right), d \geqslant 2$,

$$
H=H_{b, V}=-\Delta+b|x|^{-2}-V, \quad x \in \mathbb{R}^{d}, b \in \mathbb{R},
$$

где $V \geqslant 0$ - локально интегрируемая функция в $\mathbb{R}^{d}$. Обозначим через $N_{b}(V)$ число отрицательных собственных чисел оператора (1). Если $d \geqslant 3$ и $b>$ $-(d-2)^{2} / 4$, то справедлива оценка Цвикеля-Либа-Розенблюма (см. $\left.[4,8,11]\right)$

$$
N_{b}(V) \leqslant C(b, d) \int_{\mathbb{R}^{d}} V^{d / 2} d x .
$$

Известно также, что если $d=2$ и $b=0$, то сколь угодно малое возмущение неотрицательным потенциалом $V$ приводит к появлению по крайней мере одного отрицательного собственного числа. Это означает, что в этом случае оценка (2) невозможна.

В работах $[13,14]$, а также в работе [2] (в более сильной форме) было показано, что при некоторых дополнительных условиях на потенциал $V$ в случае $d=2$ и $b=0$ задача расщепляется на две. Первая определяется сужением оператора (1) на функции, зависящие только от $|x|$, что приводит к изучению одномерного оператора Шрёдингера. Его потенциал $\widetilde{V}$ равен среднему значению $V$ по сфере $\mathbb{S}^{1}$. В частности, для таких операторов в [3] получены необходимые и достаточные условия справедливости асимптотической формулы $N_{b=0}(\alpha \widetilde{V})=O(\alpha)$ при $\alpha \rightarrow \infty$. Вторая задача определяется классом функций, для которых среднее по сфере равно нулю. Для этого класса выполняется неравенство Харди (см. $[9,10])$, что автоматически выделяет «подпирающий» член $b|x|^{-2}$ с некоторым $b>0$. Именно этими обстоятельствами оправдывается введение члена $b|x|^{-2}, b>0$, при изучении оператора Шрёдингера в $L^{2}\left(\mathbb{R}^{2}\right)$. Заметим, однако, что «снятие» виртуального уровня в нуле выбором $b>0$ все еще не обеспечивает даже полуограниченности оператора $(1)(d=2)$, если предположить только, что $V \in L^{1}\left(\mathbb{R}^{2}\right)$.

Обозначим через $\mathscr{H}^{1}\left(\mathbb{R}^{2}\right)$ так называемое однородное пространство Соболева, определяемое равенством

$$
\mathscr{H}^{1}\left(\mathbb{R}^{2}\right)=\left\{u: \int\left(|\nabla u|^{2}+\frac{|u|^{2}}{|x|^{2}}\right) d x<\infty\right\} .
$$

Для потенциалов, зависящих от $|x|$, квадратичная форма

$$
h_{b, V}[u]=\int_{\mathbb{R}^{2}}\left(|\nabla u|^{2}+b|x|^{-2}|u|^{2}\right) d x-\int_{\mathbb{R}^{2}} V|u|^{2} d x,
$$


определенная на $\mathscr{H}^{1}\left(\mathbb{R}^{2}\right)$, полуограничена, замкнута и, следовательно, задает самосопряженный оператор $H_{b, V}$.

Нашим главным результатом является следующая теорема.

ТеоремА. Пусть $d=2, b>0 u V(x)=V(|x|) \geqslant 0$. Тогдa

¿วe

$$
N_{b}(V) \leqslant \frac{A(b)}{4 \pi} \int_{\mathbb{R}^{2}} V(x) d x,
$$

$$
A(b)=\min _{\mu>0}\left\{\mu: \mu^{-1 / 2} \cdot\left(\#\left\{n: n^{2}+b-\mu<0, n \in \mathbb{Z}\right\}\right)\right\} .
$$

ЗАМЕЧАНИЕ 1. Легко видеть, что $A(b) \rightarrow \infty$ при $b \rightarrow 0$. В частности, если $b<\mu \leqslant 1+b$, то $A(b)=\mu^{-1 / 2}$.

ЗАмЕчАНИЕ 2. Сформулированная выше теорема дает пример простого класса потенциалов, для которого верна оценка $(2)$ в случае $d=2$. По поводу обобщений этого результата см. [7].

2. Вспомогательный результат. При доказательстве теоремы мы используем предельный случай неравенства Либа-Тирринга для одномерного оператора Шрёдингера. Пусть

$$
L v(t)=-v^{\prime \prime}(t)-W(t) v(t), \quad W \geqslant 0, t \in \mathbb{R}^{1},
$$

есть самосопряженный оператор в $L^{2}\left(\mathbb{R}^{1}\right)$ с дискретным отрицательным спектром. Обозначим через $\left\{-\mu_{k}\right\}_{k=1}^{\infty}$ отрицательные собственные числа оператора $L$.

Лемма. Если при введенных выше обозначениях $W \in L^{1}\left(\mathbb{R}^{1}\right)$ u $W \geqslant 0$, mо

$$
\sum_{k} \mu_{k}^{1 / 2} \leqslant \frac{1}{2} \int W(t) d t
$$

Постоянная $1 / 2$ в правой части неравенства (6) неулучшаема, что было недавно доказано в [6] (см. также [5]). Оценка величины $\sum_{k} \mu_{k}^{1 / 2}$ через $\|W\|_{L^{1}\left(\mathbb{R}^{1}\right)}$ с некоторой постоянной, большей чем $1 / 2$, была впервые получена в [15]. Заметим, что равенство в (6) достигается в случае, когда $W(t)=\delta(t)$. При этом возникает только одно отрицательное собственное число, равное $-1 / 4$. Оба доказательства, полученные в работах [6] и [15], основаны на принципе Бирмана-Швингера [1, 12].

3. Доказательство теоремы. Рассмотрим квадратичную форму (3) и введем полярные координаты $x=(r, \theta), r \in \mathbb{R}_{+}, \theta \in[0,2 \pi]$. Тогда форма (3) перепишется в виде

$$
h_{b, V}[u]=\int_{0}^{\infty} \int_{0}^{2 \pi}\left(\left|u_{r}^{\prime}\right|^{2}+r^{-2}\left(\left|u_{\theta}^{\prime}\right|^{2}+\left(b-r^{2} V(r)\right)|u|^{2}\right)\right) r d r d \theta .
$$

Обозначим через $\left\{-\lambda_{n}\right\}$ отрицательные собственные числа оператора $H$. Тогда в силу вариационного принципа

$$
N_{b}(V)=\#\left\{n:-\lambda_{n}(H)<0\right\}=\operatorname{dim}\left\{u:(H u, u) \leqslant 0, u \in C_{0}^{\infty}\left(\mathbb{R}^{2} \backslash\{0\}\right)\right\} .
$$

Это позволяет нам работать с функциями из класса $u \in C_{0}^{\infty}\left(\mathbb{R}^{2} \backslash\{0\}\right)$. Производя замену $r=e^{t}$ и $w(t, \theta)=u\left(e^{t}, \theta\right), t \in \mathbb{R}, \theta \in[0,2 \pi]$, мы получаем, что (7) 
переходит в форму

$$
\tilde{h}[w]:=\int_{-\infty}^{\infty} \int_{\mathbb{S}^{1}}\left(\left|w_{t}^{\prime}\right|^{2}+\left(\left|w_{\theta}^{\prime}\right|^{2}+(b-\tilde{V})|w|^{2}\right)\right) d t d \theta, \quad \text { где } \tilde{V}(t)=e^{2 t} V\left(e^{t}\right) .
$$

Пусть теперь $\left\{-\mu_{k}(\widetilde{V})\right\}$ и $\left\{v_{k}(t)\right\}, k \in \mathbb{N},-$ собственные числа и собственные функции оператора $(5)$, где в качестве потенциала выбрана функция $W=\widetilde{V}$. Разделяя переменные, мы получаем, что собственные функции оператора, определяемого формой $\tilde{h}$, равны $v_{k}(t) e^{i n \theta}, n \in \mathbb{Z}, k \in \mathbb{N}$, а соответствующие собственные числа равны $b+n^{2}-\mu_{k}$. Таким образом,

$$
\begin{aligned}
N(V) & =\#\left\{(k, n): b+n^{2}-\mu_{k}, k \in \mathbb{N}, n \in \mathbb{Z}\right\} \\
& \leqslant A(b) \sum \mu_{k}^{1 / 2} \leqslant \frac{A(b)}{2} \int_{-\infty}^{\infty} \widetilde{V}(t) d t \\
& =\frac{A(b)}{2} \int_{-\infty}^{\infty} V\left(e^{t}\right) e^{2 t} d t \\
& =\frac{A(b)}{4 \pi} \int_{0}^{\infty} \int_{0}^{2 \pi} V(r) r d x \\
& =\frac{A(b)}{4 \pi} \int_{\mathbb{R}^{2}} V(x) d x,
\end{aligned}
$$

где постоянная $A(b)$ определена равенством (4). Доказательство завершено.

В заключение автор благодарит $M$. Соломяка за приглашение в институт Вейцманна (Реховот, Израиль), где автор имел возможность завершить работу над этой заметкой.

\section{ЛитЕРАТУРА}

1. Бирман М. Ш. Матем. сб., 55, 125-174 (1961). 2. Birman M. Sh., Laptev A. Comm. Pure Appl. Math., 49, 967-997 (1996). 3. Birman M. Sh., Solomyak M. Z. Adv. Soviet Math., 7, 1-55 (1991). 4. Cwikel M. Ann. Math., 106, 93-100 (1977). 5. Hundertmark D., Laptev A., Weidl T. Invent. Math., 140, 693-704 (2000). 6. Hundertmark D., Lieb E. H., Thomas L. E. Adv. Theor. Math. Phys., 2, 719-731 (1998). 7. Laptev A., Netrusov Yu. Amer. Math. Soc. Transl. Ser. 2, 189, 178-186 (1998). 8. Lieb E. H. Bull. Amer. Math. Soc., 82, 751753 (1976). 9. Maz'ya V. Sobolev Spaces. Springer-Verlag, Berlin-Heidelberg-New YorkTokyo (1985). 10. Opic B., Kufner A. Hardy-Type Inequalities. Pitman Research Notes in Mathematics Series, vol. 219, Longman Scientific \& Technical, Harlow, 1990. 11. Розенблюм Г. В. Изв. ВУЗов, сер. матем., 1, 75-86 (1976). 12. Schwinger J. Proc. Nat. Acad. Sci., 47, 122-129 (1961). 13. Solomyak M. Israel J. Math., 86, 253-276 (1994). 14. Solomyak M. Proc. London Math. Soc., 71, 53-75 (1995). 15. Weidl T. Comm. Math. Phys., 178, 135-146 (1996).

Королевская техническая школа, факультет математики, Стокгольм, Швеция 7 апреля 1999 г. laptev@math.kth.se 